\title{
Comparative Numerical Analysis of Quasi-Two-Dimensional Negative and Positive Trions in High Magnetic Fields
}

\author{
A. Trojnar and A. Wójs \\ Institute of Physics, Wrocław University of Technology \\ Wybrzeże Wyspiańskiego 27, 50-370 Wrocław, Poland
}

Exact diagonalization calculations in the Haldane spherical geometry are carried out for the three-body Coulomb problem in a quasi-two-dimensional quantum well, in the presence of perpendicular magnetic field. Simultaneous inclusion of several Landau levels and quantum well subbands in the configuration-interaction basis allowed for quantitative description of the coupling between the in-plane dynamics (governed by interplay of cyclotron quantization and Coulomb interactions) and single-particle excitations in the normal direction. The energy spectra of negative and positive trions are compared in symmetric and asymmetric wells with realistic widths and carrier concentrations. Effects of nearby impurities are also studied.

PACS numbers: 71.35.Pq, 71.35.Ji, 71.10.Pm

\section{Introduction}

In negatively doped semiconductor nanostructures, excitons $(\mathrm{X}=\mathrm{e}+\mathrm{h})$ created by photo-excitation under conditions of a typical photoluminescence (PL) experiment interact with the surrounding electron gas and easily form charged complexes called negative trions $\left(\mathrm{X}^{-}=2 \mathrm{e}+\mathrm{h}\right)$. Similarly, positive trions $\left(\mathrm{X}^{+}=\right.$ $2 \mathrm{~h}+\mathrm{e})$ form in positively doped structures through the exciton-hole capture. Dynamics of a three-body Coulomb problem in a two-dimensional (2D) quantum well of finite width $w$ (of the same order of magnitude as the excitonic Bohr radius) and in the presence of strong external magnetic field $B$ and an electric field induced by a doping (ionized donor or acceptor) layer placed on one side of the well - is fairly complicated. Competition of several energy scales (Coulomb, cyclotron, subband, and Zeeman) and coupling of the translationally invariant in-plane motion (involving Landau quantization and electrostatic electron-hole binding) with the strongly quantized single-particle motion in the normal direction make for the complexity of the trion problem. 
The combination of PL experiments [1] and numerical calculations [2] led over the years to the qualitative understanding of the trion dynamics and recombination. It has been established that the trion spectrum includes a small number of radiative ("bright") and intrinsically nonradiative ("dark") bound states, distinguished by the total (pseudo)spin $S=0$ or 1 of the pair of like carriers and the relative angular momentum $M$. The most stable trions are the bright singlet $\mathrm{X}_{\mathrm{sb}}^{ \pm}$ $(S=M=0)$ and two triplets $\mathrm{X}_{\mathrm{tb}}^{ \pm}$and $\mathrm{X}_{\mathrm{td}}^{ \pm}(S=1$ and $M=0$ and -1 for the "bright" and "dark" state, respectively).

The Coulomb binding energy of a trion is defined as the difference between the trion ground state energy and the lowest energy of an unbound (exciton plus electron) configuration, $\Delta=E_{\mathrm{X}}+E_{\mathrm{e}}-E_{\mathrm{X}^{-}}$or $E_{\mathrm{X}}+E_{\mathrm{h}}-E_{\mathrm{X}^{+}}$. The binding energy of the singlet trion must be further decreased by a Zeeman splitting $E_{\mathrm{Z}}$ of the second carrier that flips its spin when binding to the exciton. Local perturbations such as well width fluctuations [3] or nearby ionized impurities [4] can also affect trion stability and recombination.

\section{Results and discussion}

We performed large-scale exact-diagonalization calculations in the Haldane spherical geometry. Configuration-interaction bases included single-particle states from five Landau levels (LLs) and three quantum well subbands. The three-body $(2 \mathrm{e}+\mathrm{h}$ or $2 \mathrm{~h}+\mathrm{e})$ Hamiltonian matrices, counting several billion nonzero elements, were diagonalized with simultaneous resolution of spin and parity [5]. Inclusion of electron and hole excitations both in the plane of the well (intra- and inter-LL) and normal to the plane (inter-subband) allowed unprecedented accuracy of the trion energies and wave functions.

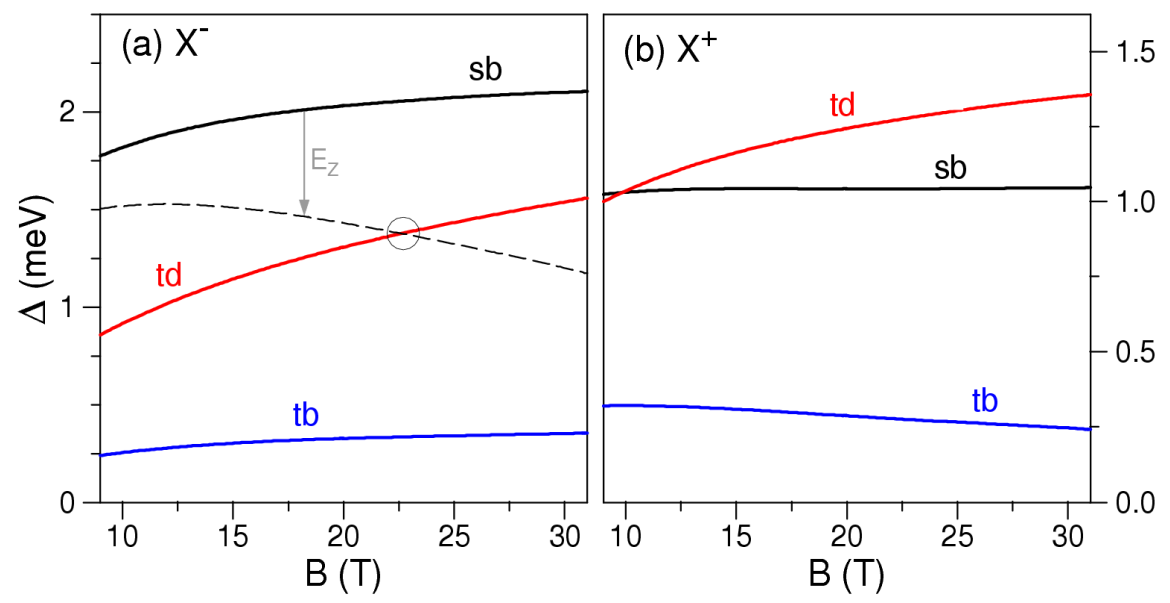

Fig. 1. Dependence of the Coulomb binding energies $\Delta$ of negative (a) and positive (b) trions on magnetic field $B$, in a symmetric GaAs well of width $w=20 \mathrm{~nm}$. Different trions are distinguished by spin $S$ and angular momentum $M$ : sb — "bright singlet" $(S=0, M=0)$, td — "dark triplet" $(1,-1)$, tb — "bright triplet" $(1,0)$. 
In Fig. 1 we present sample magnetic field dependence of the binding energy, $\Delta(B)$, for both negative and positive trions confined in a symmetric $20 \mathrm{~nm}$ GaAs quantum wells. For the $\mathrm{X}^{-}$, the bright singlet has the largest $\Delta$, but inclusion of electronic $E_{\mathrm{Z}}$ reveals a singlet-triplet crossing at $B \approx 22.5 \mathrm{~T}$. For the $\mathrm{X}^{+}$, the bright singlet is bound less strongly, and inclusion of the hole's $E_{\mathrm{Z}}$ (not shown) leads to a dark triplet ground state in the whole range of $B$. The (negative or positive) bright triplet is relatively least field dependent and least strongly bound of the three shown trions.

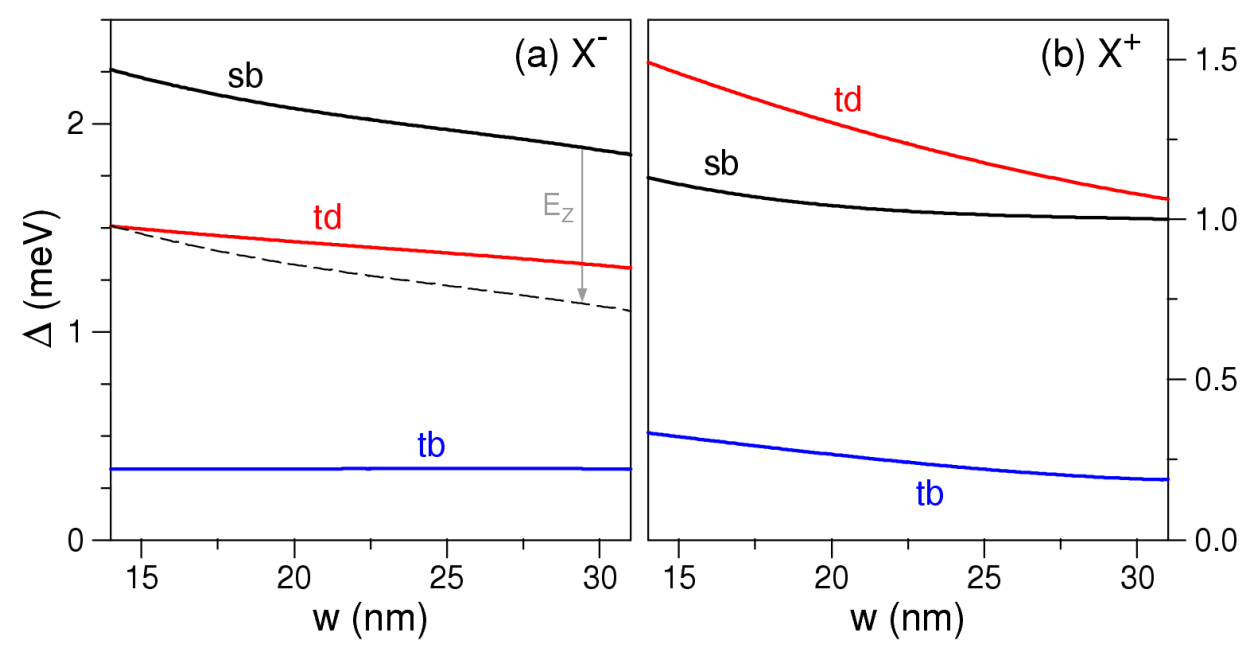

Fig. 2. The same as in Fig. 1, but with trion binding energies $\Delta$ plotted as a function of width $w$ of a symmetric GaAs quantum well in a fixed magnetic field $B=25 \mathrm{~T}$.

In Fig. 2 we show similar curves, but plotted as a function of well width, $\Delta(w)$. While the binding of most trions weakens with widening of the well, the curves for $\mathrm{X}_{\mathrm{tb}}^{-}$and $\mathrm{X}_{\mathrm{sb}}^{+}$(in wider wells) appear nearly flat.

In Fig. 3 we also show $\Delta(w)$, but for asymmetric wells, doped on one side to a typical concentration $\varrho=2 \times 10^{11} \mathrm{~cm}^{-2}$ (at the chosen high magnetic field $B=25 \mathrm{~T}$, corresponding to the LL filling factor $\nu=1 / 3$, at which a Laughlin incompressible liquid forms). The reduction of binding with increasing width is now enhanced by a quantum confined Stark shift.

In Fig. 4 we show $\Delta(B)$ for one-sided doped quantum wells, calculated at a fixed LL filling factor $\nu=1 / 3$ (i.e., at concentration $\varrho$ proportional to $B$ ). This graph could guide design of quantum wells with a desired trion ground state that could be used for an optical probe of the Laughlin liquid (it was shown [6] that only a dark triplet trion leads to discontinuities in PL at $\nu=1 / 3$ [7]). Compared to Fig. 1, the singlet-triplet crossing in the $\mathrm{X}^{-}$spectrum shifts to a slightly lower field due to the Stark effect. For $\mathrm{X}^{+}$, the dark triplet is a robust trion ground state regardless of $B, w, \varrho$, or $E_{\mathrm{Z}}$. 


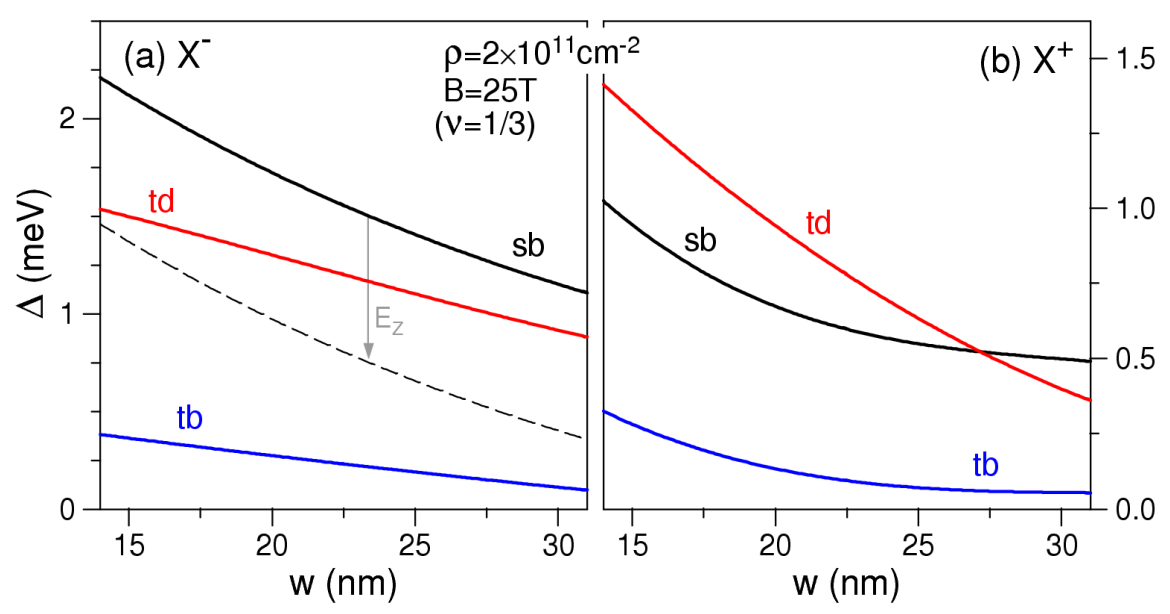

Fig. 3. Similar to Fig. 2, but for an asymmetric well, doped one side to electron or hole concentration $\varrho=2 \times 10^{11} \mathrm{~cm}^{-2}$, at magnetic field $B=25 \mathrm{~T}$, corresponding to filling factor $\nu=1 / 3$. In part (a) electron Zeeman energy is $E_{\mathrm{Z}}=0.75 \mathrm{meV}$.

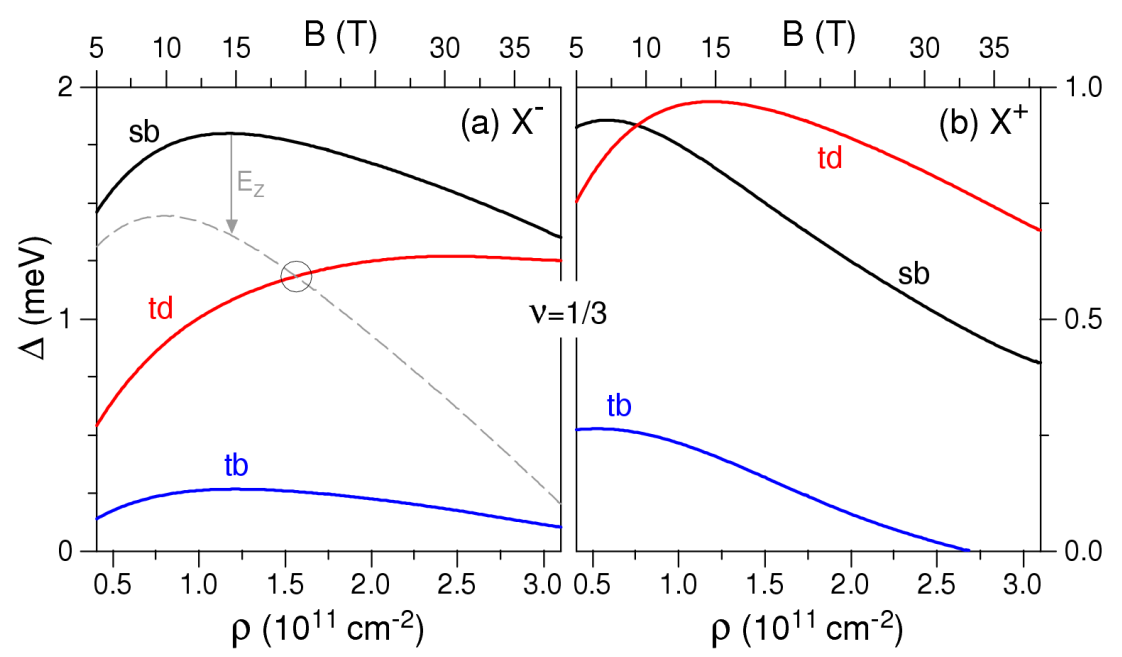

Fig. 4. Similar to Fig. 3, but with $\Delta$ plotted as a function of magnetic field $B$ and concentration $\varrho$ (proportional to each other at the fixed LL filling factor $\nu=1 / 3$ ), in a one-sided doped GaAs quantum well of width $w=20 \mathrm{~nm}$.

The reduction of all binding energies in the presence of a nearby ionized impurity is demonstrated in Fig. 1, showing $\Delta$ as a function of its distance $h$ from the well center. The binding energy is here defined as $\Delta=E_{\mathrm{X}}+E_{\mathrm{D}^{0}}-E_{\mathrm{D}^{0} \mathrm{X}}$ or $E_{\mathrm{X}}+E_{\mathrm{A}^{0}}-E_{\mathrm{A}^{0} \mathrm{X}}$, where $\mathrm{D}^{0} \mathrm{X}$ or $\mathrm{A}^{0} \mathrm{X}$ denotes an $\mathrm{X}^{-}$bound to an ionized donor or an $\mathrm{X}^{+}$bound to an ionized acceptor. Only for the bright trion states (which have $M=0)$ is this quantity equivalent to the $\mathrm{PL}$ splitting of the bound trion $\left(\mathrm{D}^{0} \mathrm{X}\right.$ 

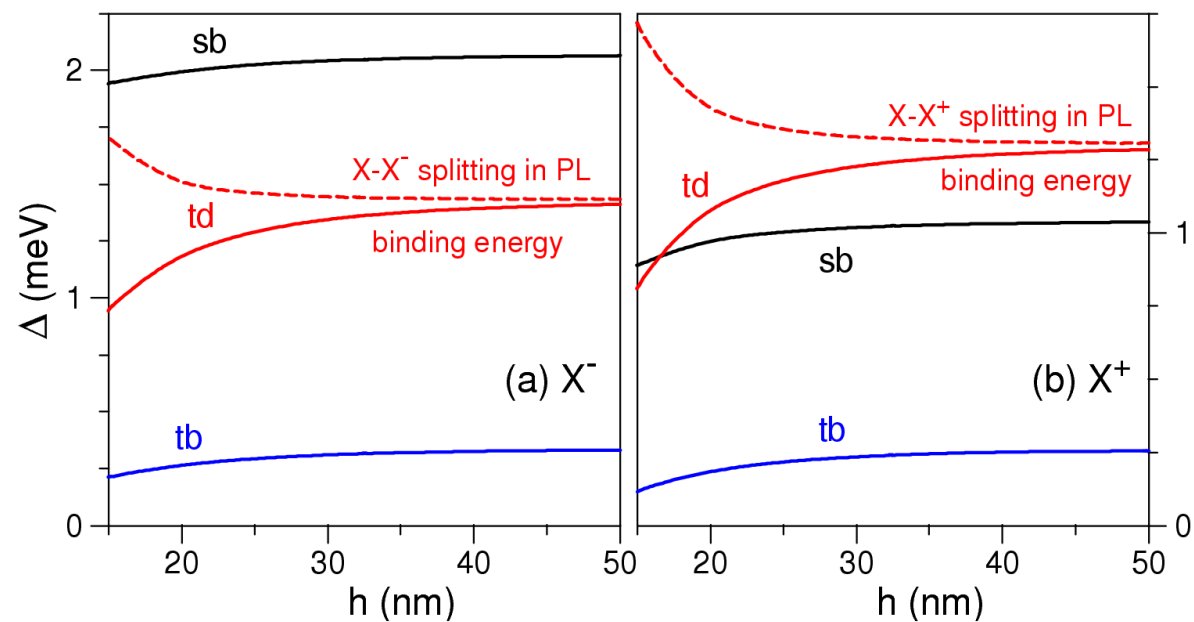

Fig. 5. Dependence of the Coulomb binding energy $\Delta$ of negative (a) and positive (b) trions on distance $h$ of a charged impurity from the center of a symmetric GaAs well of width $w=20 \mathrm{~nm}$, in magnetic field $B=25 \mathrm{~T}$. For "dark triplets" which have $M \neq 0$, binding energy $\Delta$ (solid gray line) is distinguished from the trion-exciton splitting in the PL spectrum (dashed line).
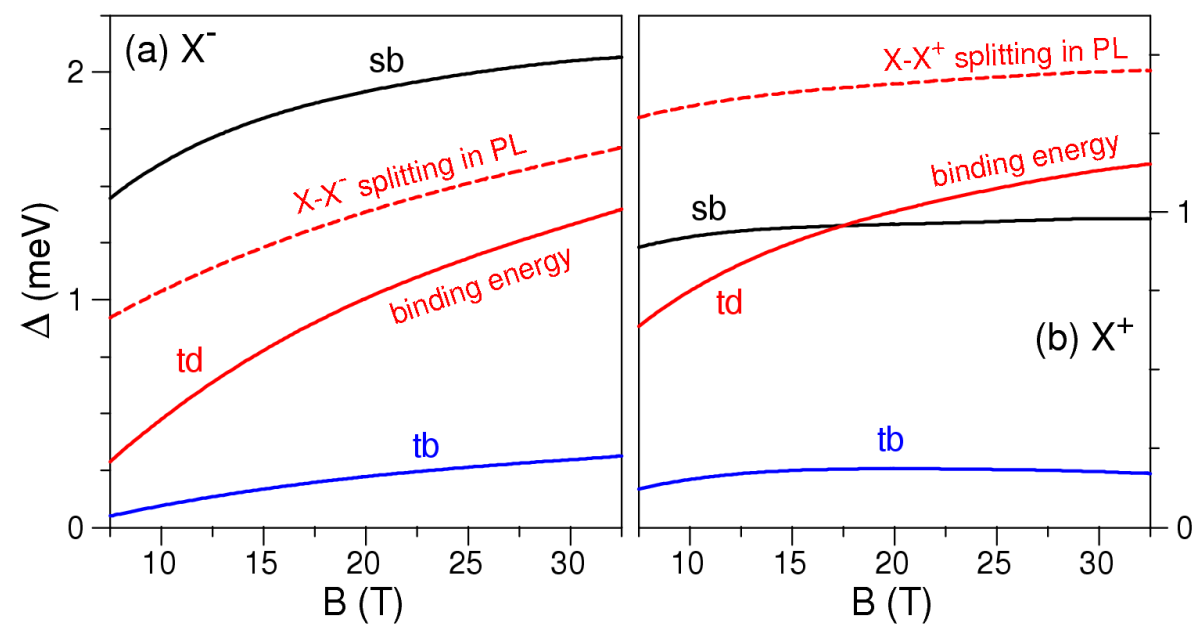

Fig. 6. Similar to Fig. 1, but in the presence of an ionized donor or acceptor at a distance $h=20 \mathrm{~nm}$ from the center of a symmetric GaAs well of width $w=20 \mathrm{~nm}$. As in Fig. 5, for the "dark triplet", binding energy (solid lines) is distinguished from the trion-exciton splitting in PL (dashed lines).

or $\mathrm{A}^{0} \mathrm{X}$ ) line from the free exciton line. For the dark triplet, the impurity-bound carrier left over after the recombination of an impurity-bound trion retains the initial angular momentum $M \neq 0$, and thus also some excitation energy. The PL 
splitting of the dark triplets, considerably higher than $\Delta$, is shown with dashed lines. In Fig. 6 we also show a sample field dependence, $\Delta(B)$, calculated in the presence of an impurity at a distance $h=20 \mathrm{~nm}$.

\section{Conclusion}

We have used exact numerical diagonalization on a Haldane sphere to investigate binding energies of trions confined in GaAs quantum wells and subjected to high magnetic fields. Different ordering of the binding energies of negative and positive trions was found for typical experimental parameters. The hypothetical sparse nearby charged impurities are shown to reduce binding energies of all trions but, at the same time, to increase the energy splitting of the dark triplet trion from the exciton line in the PL spectrum.

\section{Acknowledgments}

The authors gratefully acknowledge helpful discussions with L. Bryja and support from Polish MNiSW grant N202-071-32/1513.

\section{References}

[1] K. Kheng, R.T. Cox, Y. Merle d'Aubigne, F. Bassani, K. Saminadayar, S. Tatarenko, Phys. Rev. Lett. 71, 1752 (1993); H. Buhmann, L. Mansouri, J. Wang, P. H. Beton, N. Mori, L. Eaves, M. Henini, M. Potemski, Phys. Rev. B 51, 7969 (1995); S. Glasberg, H. Shtrikman, I. Bar-Joseph, Phys. Rev. B 63, 201308 (2001); G. Yusa, H. Shtrikman, I. Bar-Joseph, Phys. Rev. Lett. 87, 216402 (2001); D. Andronikov, V. Kochereshko, A. Platonov, T. Barrick, S.A. Crooker, G. Karczewski, Phys. Rev. B 72, 165339 (2005); L. Bryja, A. Wojs, J. Misiewicz, M. Potemski, D. Reuter, A. Wieck, Phys. Rev. B 75, 035308 (2007).

[2] B. Stebe, A. Ainane, Superlatt. Microstruct. 5, 545 (1989); A. Wójs, P. Hawrylak, Phys. Rev. B 51, 10880 (1995); D.M. Whittaker, A.J. Shields, Phys. Rev. B 56, 15185 (1997); A. Wójs, J.J. Quinn, P. Hawrylak, Phys. Rev. B 62, 4630 (2000); A.B. Dzyubenko, A.Y. Sivachenko, Phys. Rev. Lett. 84, 4429 (2000); C. Riva, F.M. Peeters, K. Varga, Phys. Rev. B 63, 115302 (2001).

[3] A.V. Filinov, C. Riva, F.M. Peeters, Yu.E. Lozovik, M. Bonitz, Phys. Rev. B 70, 035323 (2004)

[4] A.B. Dzyubenko, D.A. Cosma, A.Y. Sivachenko, Int. J. Mod. Phys. B 21, 1563 (2007).

[5] A. Wójs, J.J. Quinn, Phys. Rev. B 63, 045304 (2001); A. Wójs, J.J. Quinn, Phys. Rev. B 75, 085318 (2007); A. Wójs, Phys. Rev. B 76, 085344 (2007).

[6] A. Wójs, A. Gładysiewicz, J.J. Quinn, Phys. Rev. B 73, 235338 (2006).

[7] M. Byszewski, B. Chwalisz, D.K. Maude, M.L. Sadowski, M. Potemski, T. Saku, Y. Hirayama, S. Studenikin, D.G. Austing, A.S. Sachrajda, P. Hawrylak, Nature Phys. (London) 2, 239 (2006). 